\title{
Sferic Excitation of a Two-Layer Conducting Medium
}

\author{
Martin B. Kraichman
}

\author{
Contribution From the U.S. Naval Ordnance Laboratory, White Oak, Md.
}

(Received November 29, 1963; revised October 29, 1964)

\begin{abstract}
The tangential electric field at the surface of a horizontally stratified conducting medium of two layers is calculated for an excitation consisting of a single plane wave sferic with a slow tail. Numerical results are presented for a layer of sea water with a substrate of earth and for a layer of earth with a substrate of perfect conductivity.
\end{abstract}

\section{Introduction}

The response of a horizontally stratified, two-layer, conducting medium to a steady-state plane wave has been treated by Cagniard [1953] and Wait [1958, 1962]. Wait [1954] has also obtained results for the response of such a medium to a magnetic field step function.

In the present paper, the two-layer medium is assumed to be excited by a single plane wave sferic with a slow tail. Numerical results are presented for the tangential electric field at the surface of a layer of sea water with a substrate of earth and at the surface of a layer of earth with a perfectly conducting substrate. These results are useful in describing the influence of the substrate on the sferic noise background and on the decay of a manmade, sferic-like signal at the surface of the upper layer.

\section{Derivation of the Tangential Electric Field}

A plane wave with a time factor $\exp (j \omega t)$ is incident in air upon a horizontally stratified medium of two layers. The upper layer has a thickness $d$, an intrinsic propagation constant $\gamma_{1}$, and an intrinsic impedance $\eta_{1}$. The lower layer is of infinite thickness with the constants $\gamma_{2}$ and $\eta_{2}$. At low frequencies, the displacement current in moderately conducting ground and in sea water is negligible compared with the con- duction current. This means that the index of refraction of the upper layer is large and that the plane wave propagates in the layer in essentially a vertical direction.

Let the $x, y$ plane be the boundary between the air and the upper layer. If the tangential magnetic field $\bar{H}_{y}(0, j \omega)$ at this boundary is in the $y$-direction, the tangential electric field $\bar{E}_{x}(0, j \omega)$ at the boundary will be in the $\mathrm{x}$-direction and may be written

$$
\bar{E}_{x}(0, j \omega)=\left[\eta_{1} \frac{\eta_{2}+\eta_{1} \tanh \left(\gamma_{1} d\right)}{\eta_{1}+\eta_{2} \tanh \left(\gamma_{1} d\right)}\right] \bar{H}_{y}(0, j \omega) .
$$

This well-known expression may be derived directly from the transmission line analogy. Each layer is characterized by a permittivity $\epsilon_{i}$, a conductivity $\sigma_{i}$, and by the common permeability of free space $\mu_{0}$. Since it has been assumed that $\omega \epsilon_{i} \ll \sigma_{i}$, it follows that $\eta_{i}=\left(\frac{j \omega \mu_{0}}{\sigma_{i}}\right)^{1 / 2}$ and that $\gamma_{i}=\left(j \omega \mu_{0} \sigma_{i}\right)^{1 / 2}$. The surface impedance function $\eta(j \omega)$, given by the bracketed quantity in (1), may be rewritten in the following form:

$$
\eta(j \omega)=\left(\frac{j \omega \mu_{0}}{\sigma_{1}}\right)^{1 / 2}\left[1+2 \sum_{n=1}^{\infty}\left(\frac{1-\beta}{1+\beta}\right)^{n} e^{-2 n\left(j \omega \mu_{0} \sigma_{1}\right)^{1 / 2} d}\right],{ }_{(2)}
$$

where $\beta=\left(\frac{\sigma_{2}}{\sigma_{1}}\right)^{1 / 2}$ and $d$ is the depth of the upper layer. If the time function of the tangential magnetic field is in the form of a unit step, then the frequency spectrum 
is given by

$$
\bar{H}_{y}(0, j \omega)=\frac{1}{j \omega},
$$

and the frequency spectrum of the tangential electric field is given by

$$
\bar{E}_{x}(0, j \omega)=\frac{\eta(j \omega)}{j \omega},
$$

where $\eta(j \omega)$ is given by (2). The time function of the tangential electric field $E_{x}, s(0, t)$ corresponding to a unit step of magnetic field is then obtained from the inverse transform of (4). This transform is of the type given by Campbell and Foster [1948] as formula No. 807 and is written

$$
\begin{gathered}
E_{x, s}(0, t)=\left(\frac{\mu_{0}}{\sigma_{1}}\right)^{1 / 2}\left(\frac{1}{\pi t}\right)^{1 / 2} \\
{\left[1+2 \sum_{n=1}^{\infty}\left(\frac{1-\beta}{1+\beta}\right)^{n} e^{-n^{2} \mu_{0} \sigma_{1} d^{2} / t}\right] .}
\end{gathered}
$$

For a perfectly conducting lower layer, $\beta$ is infinite and

$$
\begin{aligned}
E_{x},{ }_{s}(0, t)= & \left(\frac{\mu_{0}}{\sigma_{1}}\right)^{1 / 2}\left(\frac{1}{\pi t}\right)^{1 / 2} \\
& {\left[1+2 \sum_{n=1}^{\infty}(-1)^{n} e^{-n^{2} \mu_{0} \sigma_{1} d^{2} / t}\right] . }
\end{aligned}
$$

Another expression for $E_{x, s}(0, t)$ when $\beta$ is infinite may be derived by obtaining the inverse Laplace transform of (4) by taking $j \omega$ as the complex variable and integrating along a semicircular contour to the left of the imaginary axis. The integration results in

$$
E_{x, s}(0, t)=\frac{2}{\sigma_{1} d} \sum_{n=0}^{\infty} \exp \left[\frac{-(2 n+1)^{2} \pi^{2}}{4 \mu_{0} \sigma_{1} d^{2}} t\right]
$$

This expression may also be derived by the direct transformation of (6) using the method described in Titchmarsh [1948]. In the case of a semi-infinite conducting medium, $d$ is infinite, and the tangential electric field at the surface is given by the first term of the expression in (5).

An application of the convolution integral to the step response in (5), (6), or (7) yields the tangential electric field $E_{x}(0, t)$ at the surface of the upper layer for an arbitrary magnetic field excitation $H_{y}(0, t)$ Thus,

$$
E_{x}(0, t)=\int_{0}^{t} E_{x, s}(0, t-\lambda) H_{y}^{\prime}(0, \lambda) d \lambda,
$$

where the prime denotes the time derivative of the magnetic field excitation.

\section{Specification of the Magnetic Field Excitation}

It is of interest to apply the above results to the case where the magnetic field is of sferic origin. The plane wave sferic chosen is characterized by an oscillatory or quasi-sinusoidal portion which appears first, and then by a slow tail portion which follows. These sferic waveforms are discussed by Hepburn [1957]; Tepley [1959]; and Wait [1962].

The choice of an analytical representation of the sferic is based on the desire to approximate commonly observed waveforms by the simplest expression possible. Accordingly, the oscillatory portion is given by

$$
\left.\begin{array}{rlrl}
H_{y}(0, t) & =0 & & \text { for } t<0, \\
& =H_{0} e^{-a t} \sin \left(\omega_{0} t-b t^{2}\right) & & \text { for } 0 \leqslant t \leqslant t_{c} \\
& =0 & & \text { for } t>t_{c},
\end{array}\right\}
$$

where

$$
\begin{aligned}
H_{0} & =3 \times 10^{-3} \mathrm{~A} / \mathrm{m}, \\
a & =2.9 \times 10^{3} \mathrm{sec}^{-1}, \\
b & =1.8 \times 10^{7} \mathrm{sec}^{-2}, \\
\omega_{0} & =5.2 \times 10^{4} \mathrm{sec}^{-1}, \text { and } \\
t_{c} & =8.6 \times 10^{-4} \mathrm{sec} .
\end{aligned}
$$

A plot of the oscillatory waveform described by (9) is shown in figure 1 for times greater than $10^{-4} \mathrm{sec}$. The slow tail following the oscillatory portion of the sferic is represented as a positive half cycle consisting

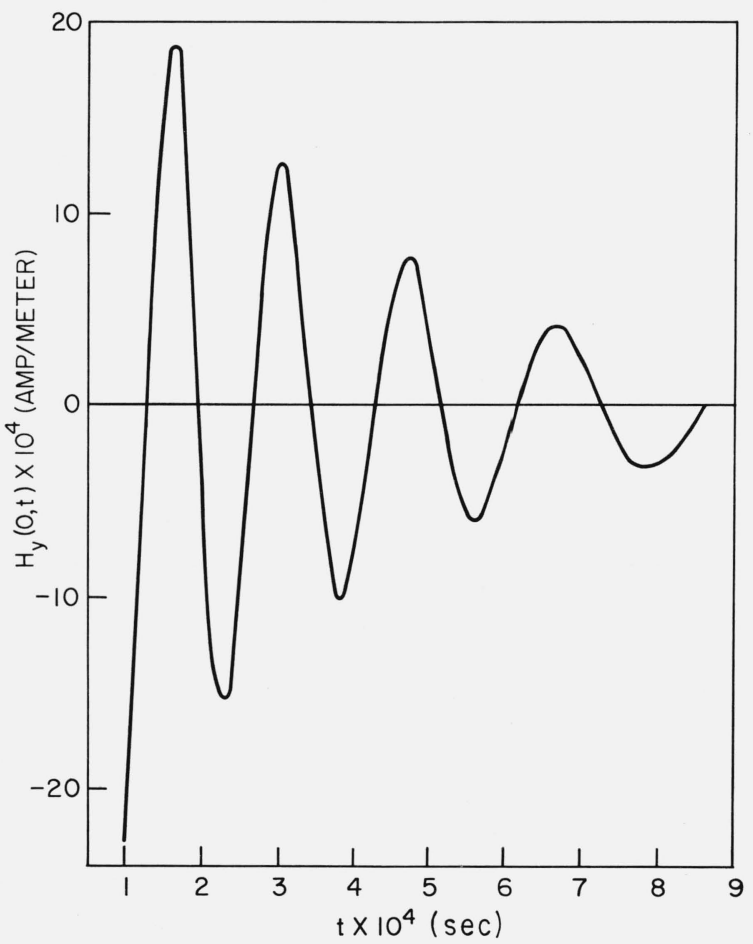

FIGURE 1. Oscillatory portion of the sferic excitation. 


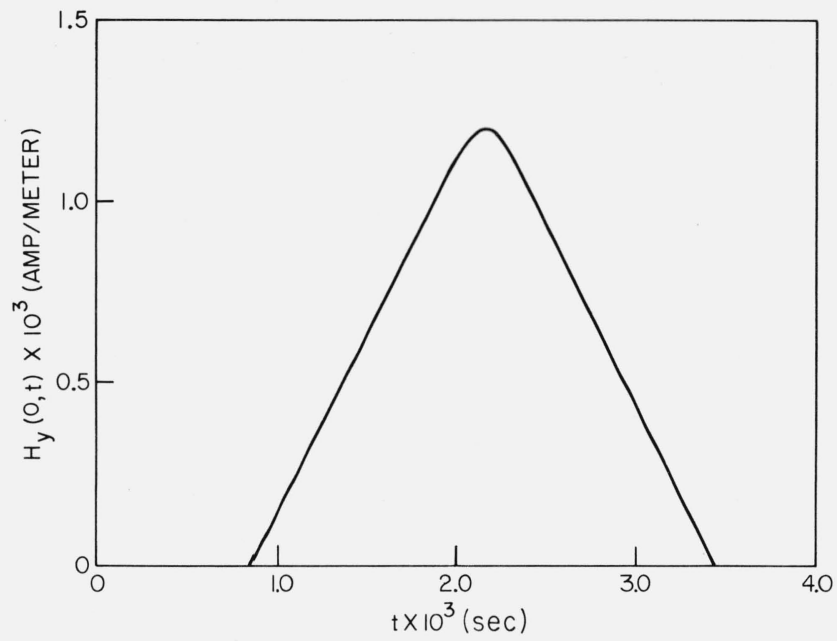

FIGURE 2. Slow tail portion of the sferic excitation.
A plot of the tangential electric field at the surface of the layer of sea water is presented in figure 3 for times from $10^{-4} \mathrm{sec}$ up to the onset of the slow tail. At these times, $t<<\mu_{0} \sigma_{1} d^{2}$, so that the step response for a layer of sea water of finite depth is equivalent to that for a layer of infinite depth. This means that the presence of the substrate will not noticeably change the value of the electric field at the sea surface and that figure 3 represents the response of a semi-infinite ocean as well as the response of a sea water layer of finite depth. Figure 4 extends the plot of the electric field to just beyond the cutoff of the slow tail. In this figure, a small difference is discernible between the response of the semi-infinite ocean and that of a layer $30 \mathrm{~m}$ deep. Figure 5 shows the electric field decay for times out to $10 \mathrm{sec}$ for the semi-infinite ocean and for layers $30 \mathrm{~m}$ and $150 \mathrm{~m}$ deep. For the times indicated and for those beyond, all the fields remain negative and decay towards zero. The rate of decay of these fields for large $t$ may be determined analytically from the step response in (5) and the convolution integral in (8). It is found that all fall off as $t^{-3 / 2}$ for large $t$. of two straight line portions joined by a section of a parabola. The duration of the slow tail is three times that of the oscillatory portion and the peak amplitude is 0.4 that of the maximum oscillatory amplitude. Thus,

$$
\left.\begin{array}{rlrl}
H_{y}(0, t) & =\alpha\left(t-t_{c}\right) & & \text { for } t_{c} \leqslant t \leqslant t_{1} \\
& =0.4 H_{0}-\beta\left(t-2.5 t_{c}\right)^{2} & & \text { for } t_{1}<t<t_{2}, \\
& =-\alpha\left(t-t_{f}\right) & & \text { for } t_{2} \leqslant t \leqslant t_{f} \\
& =0 & & \text { for } t>t_{f}
\end{array}\right\}
$$

where

and

$$
\begin{aligned}
t_{1} & =2.3 t_{c}, \\
t_{2} & =2.7 t_{c}, \\
t_{f} & =4 t_{c}, \\
\alpha & =\frac{0.4 H_{0}}{1.4 t_{c}}
\end{aligned}
$$

$$
\beta=\frac{H_{0}}{1.4 t_{c}^{2}} \text {. }
$$

A plot of the above slow tail is shown in figure 2.

\section{Numerical Results for the Sea Water and Earth Medium}

Using the sferic excitation described above, the tangential electric field at the surface of a layer of sea water with a substrate of earth has been calculated with the help of a high speed digital computer. The step response in (5) and the convolution integral in (8) were used in making the calculations. The values of the parameters in these equations are

$\sigma_{1}=$ conductivity of sea water $=4 \mathrm{mho} / \mathrm{m}$,

$\sigma_{2}=$ conductivity of earth substrate $=10^{-3} \mathrm{mho} / \mathrm{m}$,

$\mu_{0}=$ permeability of free space $=4 \pi \times 10^{-7} \mathrm{H} / \mathrm{m}$, and

$d=$ thickness of sea water layer $=30 \mathrm{~m}$ and $150 \mathrm{~m}$.

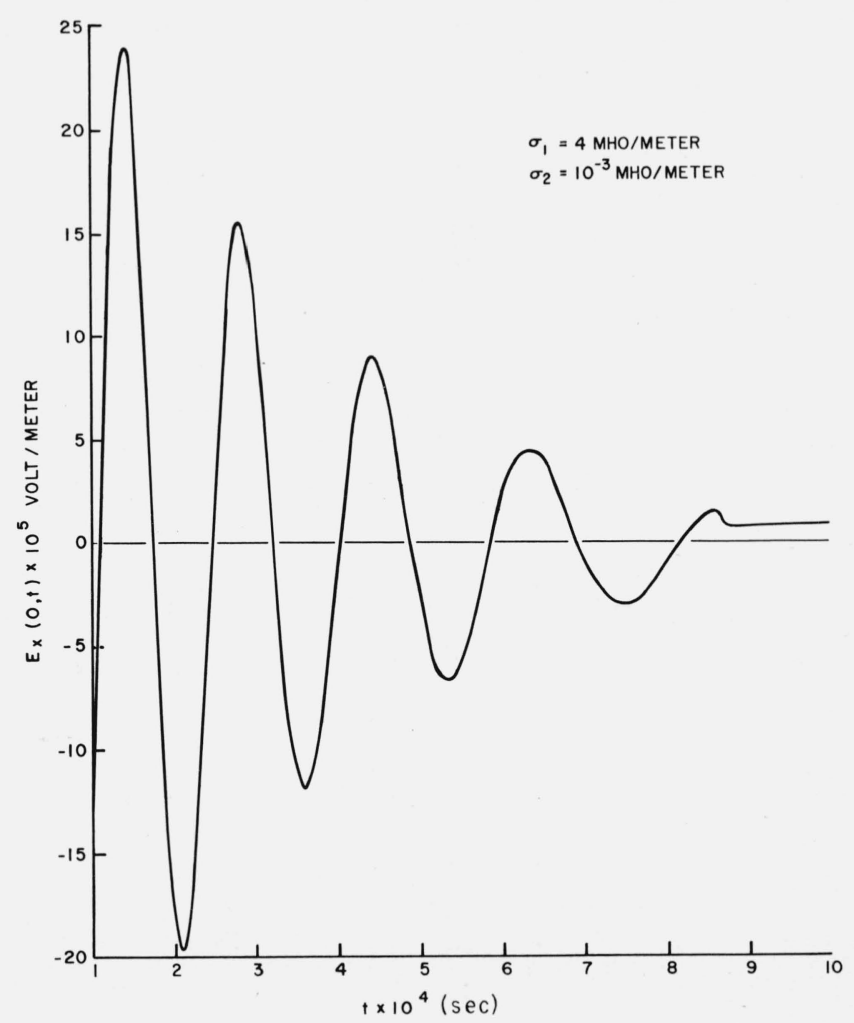

FIGURE 3. Electric field at the sea water surface during the oscillatory excitation. 


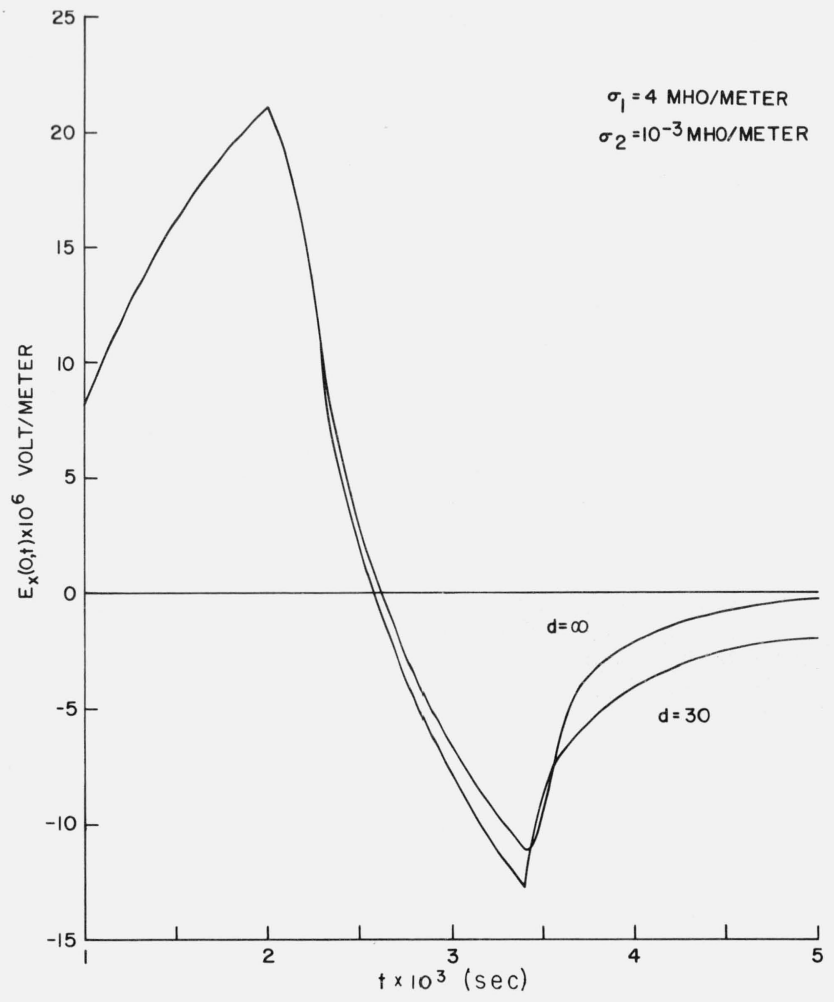

FIGURE 4. Electric field at the sea water surface during the slow tail excitation.

\section{Numerical Results for the Earth and Perfect Conductor Medium}

The response of a layer of earth with a perfectly conducting substrate is quite different from that of a sea water-earth medium. The nature of the tangential electric field at the surface of the earth layer may best be determined by an examination of the step response given in (7) and its contribution to the convolution integral in (8). The values of the parameters in these equations are

$\sigma_{1}=$ conductivity of earth layer $=10^{-3} \mathrm{mho} / \mathrm{m}$,

$\mu_{0}=$ permeability of free space $=4 \pi \times 10^{-7} \mathrm{H} / \mathrm{m}$, and

$d=$ thickness of earth layer $=30 \mathrm{~m}$.

For these values, the terms of the exponential series for the step response in (7) are extremely small when $t>10^{-6}$ sec. Since the times of interest in this paper are greater than a microsecond, the expression for the electric field in (8) may be written

$E_{x}(0, t) \approx \frac{2}{\sigma_{1} d} H_{y}^{\prime}(0, t) \int_{0}^{t} \sum_{n=0}^{\infty} \exp \left[\frac{-(2 n+1)^{2} \pi^{2}}{4 \mu_{0} \sigma_{1} d^{2}}(t-\lambda)\right] d \lambda$.

In the above expression, the time derivative of the magnetic field is taken outside the integral since the

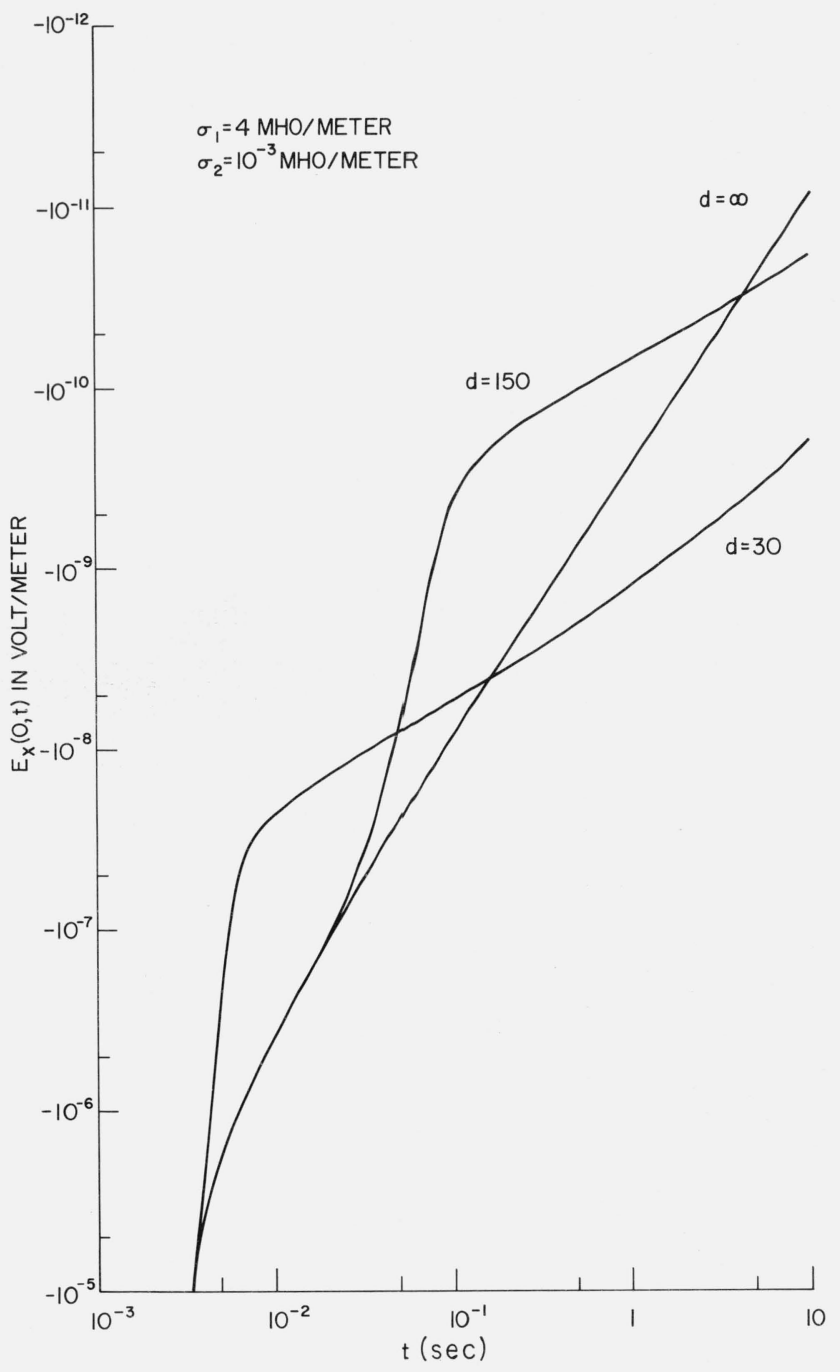

FiguRE 5. Electric field decay at the sea water surface after the cutoff of the sferic excitation.

derivative is practically constant over the small range of $\lambda$ near $t$ for which the step response contributes significantly. This means that the electric field $E_{x}(0, t)$ is proportional to the time derivative of the magnetic field excitation and that when this excitation cuts off, so almost instantaneously does $E_{x}(0, t)$.

A plot of the tangential electric field at the surface of the layer of earth is presented in figure 6 for times from $10^{-4}$ sec up to the onset of the slow tail. Results for both an infinite thickness of earth and a thickness of $30 \mathrm{~m}$ are shown in the figure. The behavior predicted by (11) is shown in figure 7 for $d=30 \mathrm{~m}$. It is observed that when the magnetic field slow tail excitation stops, the electric field almost instantaneously goes to zero. The behavior of the response of the infinitely thick layer of earth is similar to that of the semi-infinite sea water medium. 


\section{References}

Cagniard, L. (1953), Basic theory of the magneto-telluric method of geophysical prospecting, Geophysics 18, 605-635.

Campbell, G. A. and Foster, R. M. (1948), Fourier integrals for practical applications, p. 93 (D. Van Nostrand Co., Inc., New York, N.Y.).

Hepburn, F. (1957), Atmospheric waveforms with very low-frequency components below $1 \mathrm{kc} / \mathrm{s}$ known as slow tails, J. Atmospheric Terrest. Phys. 10, 266-287.

Tepley, L. R. (Dec. 1959), A comparison of sferics as observed in the very low frequency and extremely low frequency bands, J. Geophys. Res. 64. No. 12, 2315-2329.
Titchmarsh, E. C. (1948), Introduction to the theory of Fourier integrals, ch. II, 60-65 (Oxford University Press, London).

Wait, J. R. (1954), On the relation between telluric currents and the earth's magnetic field, Geophysics, 19, 281-289.

Wait, J. R. (1958), Transmission and reflection of electromagnetic waves in the presence of stratified media, J. Res. NBS 61, No. 3, 205-232, RP2899.

Wait, J. R. (1962), Electromagnetic waves in stratified media, ch. II, 53-61 (Macmillan Co., New York).

(Paper 69D3-480) 